\title{
Molecular-Dynamics Simulations of C- and N-Terminal Peptide Derivatives of GCN4-p1 in Aqueous Solution
}

\author{
by John H. Missimer*a ), Michel O. Steinmetz ${ }^{a}$ ), Wolfgang Jahnke ${ }^{\text {b }}$, Fritz K. Winkler ${ }^{a}$ ), \\ Wilfred F. van Gunsteren ${ }^{c}$ ), and Xavier Daura $\left.{ }^{c}\right)^{\text {, }}$ ) \\ a) Biomolecular Research, Paul Scherrer Institut, CH-5232 Villigen (e-mail: john.missimer@psi.ch) \\ b) Novartis Institute for Biomedical Research, Discovery Technologies, CH-4002 Basel \\ c) Laboratory of Physical Chemistry, Swiss Federal Institute of Technology Zurich, ETH-Hönggerberg, \\ CH-8093 Zurich \\ d) Catalan Institution for Research and Advanced Studies (ICREA) and Institute of Biotechnology and \\ Biomedicine, Universitat Autònoma de Barcelona, E-08193 Bellaterra
}

\begin{abstract}
We report the investigation of two 16-residue peptides in aqueous solution by means of molecular-dynamics simulations. The peptides constitute the $\mathrm{C}$ - and N-terminal halves of the 33-residue monomer whose dimer constitutes the leucine zipper of the yeast transcriptional activator, denoted GCN4-p1. To examine a hypothesis about coiled-coil formation, in which the $\mathrm{C}$-terminal half contains a helix-formation trigger site absent in the $\mathrm{N}$ terminal half, experimental studies of the two peptides have determined their helix propensities under several conditions of temperature, $\mathrm{pH}$, and salt concentration with circular dichroism. An NMR experiment provides additional evidence. At temperatures of 278 and $325 \mathrm{~K}$ and $\mathrm{pH} 7.5$, mixtures of $\alpha$ - and $\pi$-helical secondary structure constitute the most probable conformations in both $\mathrm{C}$ - and $\mathrm{N}$-terminal halves. A bifurcated salt bridge between Arg25 and Glu22/20 correlates with the structural fluctuations of the C-terminal half. It also exhibits a persistent loop at the N-terminal end involving the side chains of His18 and Glu22, which is reminiscent of helixcapping boxes. Nonreversible unfolding appears to occur abruptly in the Arg25 mutant, suggesting a cooperative event. Analysis does not indicate that the N-terminal half is less stable than the C-terminal half, indicating that $100 \mathrm{~ns}$ is too short a period to observe complete unfolding.
\end{abstract}

Introduction. - Molecular-dynamics (MD) simulation has proven to be a useful tool to study peptide folding in solution under reversible conditions at atomic resolution and to test models of the folding process [1-19]. MD Simulation of peptide stability and folding is currently an area of intense activity, and computational studies on helixforming peptides have been recently reported [1-7].

Coiled coils represent a compelling challenge for MD simulations, because we still lack detailed insight into the molecular-folding mechanisms of this universal protein subunit oligomerization motif. One of the most widely characterized coiled coils is the parallel two-stranded 33-residue leucine zipper of the yeast transcriptional activator GCN4, denoted GCN4-p1 [20]. Biophysical analyses of two monomeric 16-residue peptides derived from GCN4-p1 indicated that the C-terminal half displayed a marked propensity to form a stable helix in aqueous solution [21]. In contrast, the N-terminal half displayed only marginal, if any, propensity. These properties support the hypothesis that the C-terminal half constitutes a trigger sequence, an autonomous helical folding unit that drives coiled-coil formation by significantly limiting the number of possible chain conformations prior to the encounter of two monomers [22-24]. 
To examine this hypothesis, we performed MD simulations of the C- and Nterminal halves of the monomer. The experimental studies have determined their stability under several conditions of temperature, $\mathrm{pH}$, and salt concentration by using circular dichroism (CD) spectroscopy. An NMR experiment at $278 \mathrm{~K}$ provides additional structural information. The $\alpha$-helical structures from which dynamics of the monomeric peptides began were deduced from the X-ray crystallographic model of the leucine zipper GCN4-p1 [20].

Although MD simulations of a biomolecular system may yield structural, energetic, and dynamic information at the atomic level, which is often inaccessible to experimental probes, its predictive power is still limited by two factors: $i$ ) the quality of the atomic interaction or force field used, and $i$ ) the available computing power, which restricts the size of the system and the length of time that can be simulated [25]. The larger a biomolecular system, the longer the relaxation time for a particular physical observable may be, which may impair proper equilibration and sampling of a property of interest [26]. For example, the stability of secondary-structure elements or molecular complexes can be probed with simulations on the nanosecond time scale, whereas proper statistical averages for folding processes may require hundreds of nanoseconds. Omitting solvent degrees of freedom can accelerate peptide simulations. However, we chose to explicitly include the solvent, i.e., $\mathrm{H}_{2} \mathrm{O}$, in the simulations to realistically represent dielectric, entropic, temperature, and co-solvent effects in the simulations. The application of MD simulation techniques to study stability and formation of coiled coils is at the frontier of its use in terms of convergence, statistics, and accuracy of results. Therefore, we present properties of interest as functions of time, which allows for an assessment of convergence and statistics.

The leucine zipper dimer consists of two identical 33 amino acid monomers with the sequence: R-MKQLEDK-VEELLSK-NYHLENE-VARLKKL-VGER; the dashes separate the heptad repeats, which are fundamental patterns of coiled coils [27]. The C-terminal half, denoted by GCN4p16-31, is defined by the sequence: Ac-NYHLENEVARLKKLV-G-CONH${ }_{2}$, where the acetyl head group, Ac, and the amide tail group, $\mathrm{CONH}_{2}$, are indicated explicitly. The mutant GCN4p16-31R25G is obtained by replacing Arg25 by glycine. The N-terminal half, denoted by GCN4p2-17, is defined by the sequence: Ac-MKQLEDK-VEELLSK-NY-CONH 2 . For simulations of the peptides at $\mathrm{pH} 7.5$, histidine was singly protonated at NE2; arginine and lysine were protonated. $\mathrm{pH} 3$ was modeled by assuming the protonated forms of glutamic acid and aspartic acid and the doubly protonated form of histidine.

The results of eight MD simulations are presented: GCN4p16-31 starting from an $\alpha$ helical structure at temperatures of 278 (50 ns) and $325 \mathrm{~K}(100 \mathrm{~ns})$, and $\mathrm{pH} 7.5$, at $278 \mathrm{~K}$ and $\mathrm{pH} 3(50 \mathrm{~ns})$, and at $278 \mathrm{~K}$ in $450 \mathrm{~mm} \mathrm{NaCl}$ (50 ns). Furthermore, simulations of GCN4p16-31 were performed at $278 \mathrm{~K}$ and $\mathrm{pH} 7.5$ starting from an extended structure (100 ns), and of a mutant, R25G, of GCN4p16-31 at $325 \mathrm{~K}$ and pH 7.5 (68 ns). Simulations were performed of GCN4p2-17 at 278 K (50 ns) and $325 \mathrm{~K}$ (100 ns) and $\mathrm{pH} 7.5$ starting from an $\alpha$-helical structure.

Results. - Results of the NMR experiments are presented first, followed by the results of the eight MD simulations, which are summarized in Table 1. 
Table 1. Summary of the Simulations Performed

\begin{tabular}{|c|c|c|c|c|c|c|c|}
\hline$T[\mathrm{~K}]$ & $\mathrm{pH}-$ Salt & Initial structure & $\begin{array}{l}\text { Number of } \\
\text { atoms }\end{array}$ & $\begin{array}{l}\text { Number of } \mathrm{H}_{2} \mathrm{O} \\
\text { molecules }\end{array}$ & $\begin{array}{l}\text { Box size } \\
{\left[\mathrm{nm}^{3}\right]}\end{array}$ & Ions & $\begin{array}{l}\text { Sim period } \\
\text { [ns] }\end{array}$ \\
\hline \multicolumn{8}{|c|}{ GCN4p16-31 } \\
\hline 278 & 7.5 & $\alpha$-helix & 175 & 2977 & 91 & & 50 \\
\hline 325 & 7.5 & $\alpha$-helix & 175 & 2977 & 91 & & 100 \\
\hline 278 & 3 & $\alpha$-helix & 178 & 2973 & 91 & $4 \mathrm{Cl}$ & 50 \\
\hline 278 & $\begin{array}{l}7.5 \\
450 \mathrm{~mm}\end{array}$ & $\alpha$-helix & 175 & 2897 & 91 & $\begin{array}{l}25 \mathrm{Na} \\
+25 \mathrm{Cl}\end{array}$ & 50 \\
\hline 278 & 7.5 & extended & 175 & 8183 & 247 & & 100 \\
\hline 325 & 7.5 & $\begin{array}{l}\alpha-\pi \text {-helix } \\
\text { R25G mut }\end{array}$ & 175 & 2977 & 91 & & 68 \\
\hline \multicolumn{8}{|c|}{ GCN4p2-17 } \\
\hline 278 & 7.5 & $\alpha$-helix & 177 & 2848 & 88 & & 50 \\
\hline 325 & 7.5 & $\alpha$-helix & 177 & 2848 & 88 & & 100 \\
\hline
\end{tabular}

NMR. Analysis of the NMR experiments performed on the C-terminal half of GCN4-p1 at $278 \mathrm{~K}$ revealed for some residues NOEs, $\mathrm{H}_{\mathrm{N}}(i)-\mathrm{H}_{\mathrm{N}}(i+1)$, typical of helical structures. No $\mathrm{H}_{\mathrm{N}}(i)-\mathrm{H}_{\mathrm{N}}(i+3)$ NOEs were obtained. The lack of concentration dependence of the NMR intensities on dilution (not shown) indicates that helix formation by the peptide is a monomolecular process and not the result of aggregation. This conclusion is furthermore supported by concentration-dependent CD measurements [28]. As Table 2 shows, the helical propensity is not uniform along the sequence. Judged by the intensities of $\mathrm{H}_{\mathrm{N}}(i)-\mathrm{H}_{\mathrm{N}}(i+1)$ NOEs, substantial helical structure is evident beyond Asn21 in the C-terminal part of GCN4p16-31, but little helical structure is observed for the N-terminal residues preceding Glu22. The characteristic

Table 2. Measured and Simulated HN(i)-HN(i + 1) NOEs of GCN4p16-31 in Aqueous Solution. Measured with $3 \mathrm{~mm}$ peptide, $10 \mathrm{~mm} \mathrm{NaCl}$, and $20 \mathrm{~mm} \mathrm{Na}$-phosphate at pH 7.4 and $278 \mathrm{~K}$ using NOESY with a mixing time of $150 \mathrm{~ms}$. Simulated values of $\left\langle r^{-6}\right\rangle^{-1 / 6}$ at $278 \mathrm{~K}$ and $\mathrm{pH}$ 7.5. $\left.\delta<r^{-6}\right\rangle^{-1 / 6}$ denotes the standard deviation about the mean.

\begin{tabular}{|c|c|c|c|c|}
\hline \multicolumn{2}{|c|}{${ }^{1} \mathrm{H}$-Resonance } & \multirow{2}{*}{$\begin{array}{l}\text { NOE intensity } \\
\text { [arbitrary units] }\end{array}$} & \multicolumn{2}{|l|}{$\underline{\text { Simulation }}$} \\
\hline Residue $i$ & Residue $i+1$ & & $<r^{-6}>^{-1 / 6}[\mathrm{~nm}]$ & $\left.\delta<r^{-6}\right\rangle^{-1 / 6}[\mathrm{~nm}]$ \\
\hline 16ASN & 17TYR & 2 & 0.356 & 0.072 \\
\hline 17TYR & $18 \mathrm{HIS}$ & 3 & 0.283 & 0.030 \\
\hline $18 \mathrm{HIS}$ & 19LEU & 5 & 0.296 & 0.029 \\
\hline 19LEU & $20 \mathrm{GLU}$ & 18 & 0.286 & 0.029 \\
\hline $20 \mathrm{GLU}$ & $21 \mathrm{ASN}$ & 11 & 0.277 & 0.031 \\
\hline $21 \mathrm{ASN}$ & $22 \mathrm{GLU}$ & 10 & 0.288 & 0.030 \\
\hline $22 \mathrm{GLU}$ & $23 \mathrm{VAL}$ & 31 & 0.289 & 0.026 \\
\hline $23 \mathrm{VAL}$ & 24ALA & 36 & 0.298 & 0.025 \\
\hline 24ALA & $25 \mathrm{ARG}$ & 22 & 0.296 & 0.025 \\
\hline $25 \mathrm{ARG}$ & 26LEU & 23 & 0.289 & 0.026 \\
\hline 26LEU & 27LYS & 24 & 0.288 & 0.027 \\
\hline 27LYS & 28LYS & 19 & 0.283 & 0.026 \\
\hline 28LYS & 29LEU & 20 & 0.280 & 0.037 \\
\hline 29LEU & $30 \mathrm{VAL}$ & 22 & 0.277 & 0.058 \\
\hline $30 \mathrm{VAL}$ & 31GLY & 5 & 0.288 & 0.099 \\
\hline
\end{tabular}


asymmetric distribution of helix structure towards the C-terminus of GCN4p16-31 is further supported by the analysis of secondary $\mathrm{H}_{\alpha}$ and $\mathrm{C}_{\alpha}$ chemical shifts (not shown) and the ${ }^{3} J\left(\mathrm{H}_{\mathrm{N}}, \mathrm{H}_{\mathrm{C}_{a}}\right)$ coupling constants displayed in Table 3. The NOESY spectrum is available as Supplementary Material $\left.^{1}\right)$.

Table 3. ${ }^{3} \mathrm{~J}\left(\mathrm{H}_{N}, H_{C \alpha}\right)$ Coupling Constants [Hz] Observed for GCN4p16-31 in Aqueous Solution. Conditions of measurement and simulation as in Table 2.

\begin{tabular}{lllll}
\hline Residue & Experiment & Random coil [29] & Simulation & Simulation uncertainty \\
\hline 16ASN & 7.3 & 7.4 & 6.7 & 2.4 \\
17TYR & 6.4 & 7.3 & 4.3 & 1.4 \\
18 HIS & 7.1 & 7.2 & 4.6 & 1.6 \\
19LEU & 5.9 & 7.1 & 4.9 & 1.6 \\
20GLU & 5.1 & 6.8 & 5.8 & 1.8 \\
21ASN & 6.7 & 7.4 & 5.6 & 1.9 \\
22GLU & 5.6 & 6.8 & 5.2 & 1.7 \\
23VAL & 5.2 & 7.7 & 5.2 & 1.5 \\
24ALA & 5.5 & 6.1 & 4.8 & 1.4 \\
25ARG & 5.2 & 7.2 & 5.4 & 1.8 \\
26LEU & 5.6 & 7.1 & 5.6 & 1.9 \\
27LYS & 6.8 & 7.1 & 5.3 & 2.0 \\
28LYS & 6.5 & 7.1 & 6.1 & 2.1 \\
29LEU & 6.9 & 7.1 & 6.4 & 1.9 \\
30VAL & 6.8 & 7.7 & 7.8 & \\
\hline
\end{tabular}

GCN4p16-31 at $p H 7.5$. At both temperatures, the peptide passes from the initial pure $\alpha$-helical configuration within 10 ns into mixed conformations containing $\alpha$-and $\pi$-helical secondary structure. The simulation at $278 \mathrm{~K}$ then develops markedly differently from that at $325 \mathrm{~K}$, as a comparison of Figs. 1 and 2 shows. The top left panels of the figures display the evolution of secondary structure as determined by the program PROCHECK [30], the lower left panels, the evolution of the atom-positional root-mean-square deviations (RMSD) from the initial configuration of backbone atoms. The top right panels show the cumulative distributions of helical propensity of each residue derived from the secondary structure, and the lower right panels the cumulative distribution of the RMSD values. The secondary structures represented in Fig. 1 reveal that the conformations following the initial $\alpha$-helical configuration at $278 \mathrm{~K}$ are characterized by a combination of $\alpha$ - and $\pi$-helices in all residues between Tyr17 and Val30, with a substantial proportion of $\alpha$-helical structure in residues between Tyr17 and Glu20. The helical structures in the N-terminal residues Tyr17 and His 18 disappear at $325 \mathrm{~K}$. Fig. 2 evidences two regions of residues following the initial $\alpha$-helical configuration: residues Asn16 to His18 possess no secondary structure, whereas residues Leu19 to Asn21 evidence $\pi$-helices and H-bonded turns. At both temperatures, $\pi$-helical conformations dominate in the residues from Asn21 to Val30. The peaks of the RMSD distributions differ by $c a .0 .03 \mathrm{~nm}$ for the two temperatures, the smaller value for $278 \mathrm{~K}$ reflecting the greater helical propensity in the first residues. Evident in the RMSD distributions at both temperatures are excursions to deviations larger than the peak values, which appear associated with the dissolution of helical

1) Supplementary Material is available upon request from the corresponding author. 


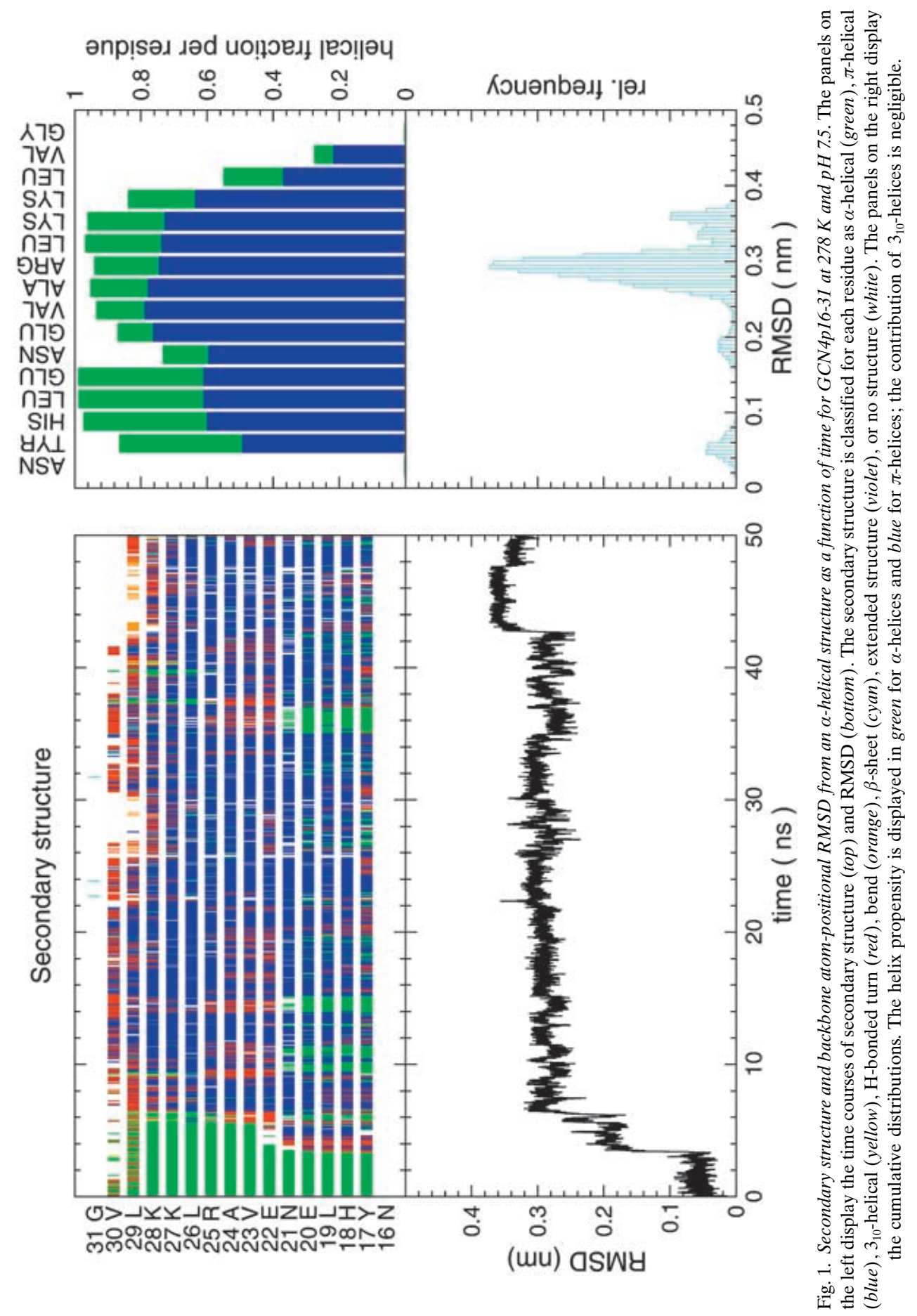




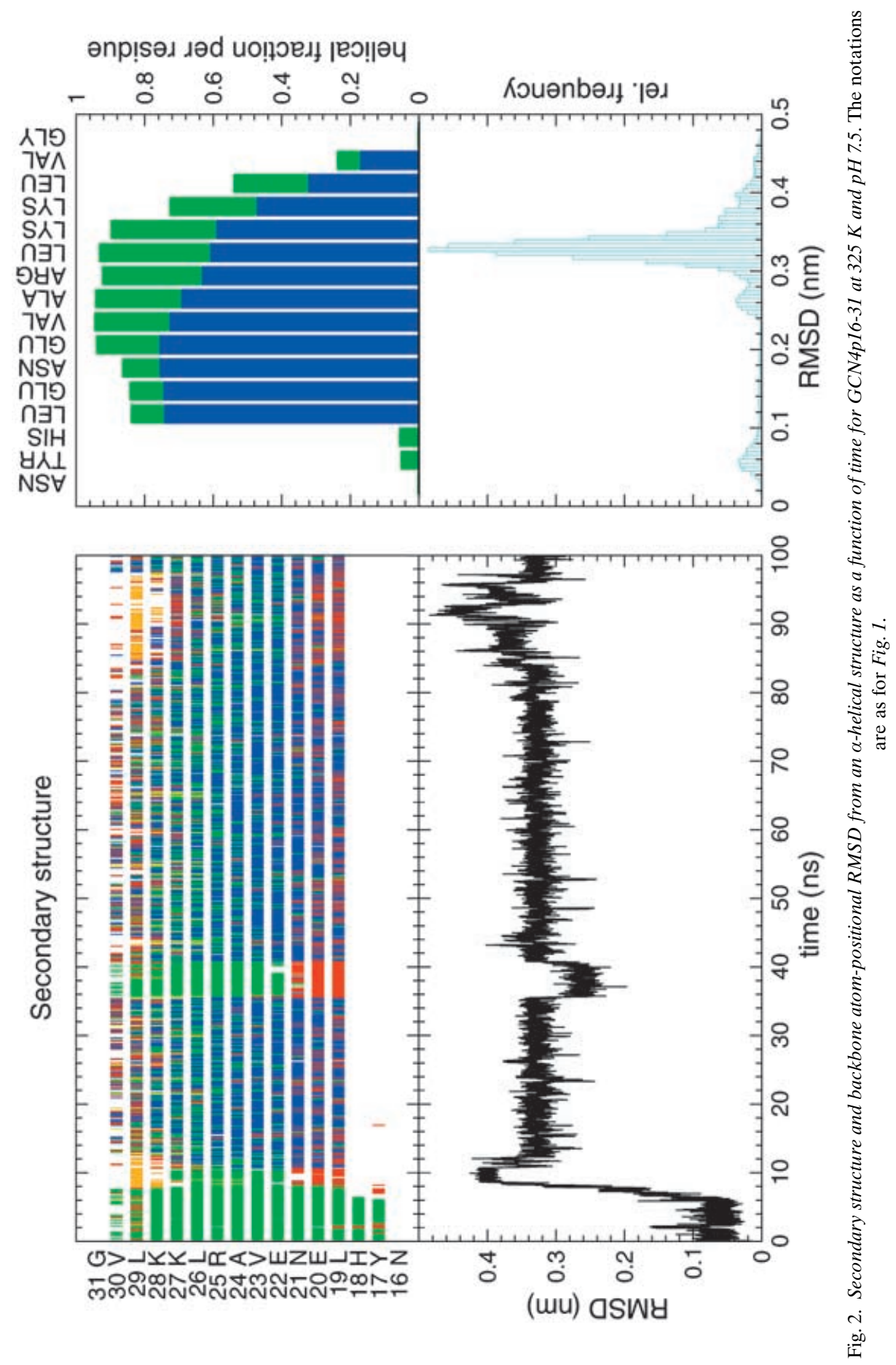


secondary structure in residues Lys28, Leu29, and Val30. Analysis of the H-bonds, for which the time courses of the peptide at $325 \mathrm{~K}$ are shown in the upper panel of Fig. 3, reflects the dominance of $\pi$-helices in the intermediate residues. $i, i+5$ bonds between backbone atoms characteristic of $\pi$-helices occur with frequencies between 75 and $85 \%$ at $278 \mathrm{~K}$ and between 65 and $80 \%$ at $325 \mathrm{~K} . i, i+4$ Bonds between backbone atoms characteristic of $\alpha$-helices occur with frequencies between 10 and $20 \%$. Analyses of atom-positional RMSD, secondary structure and H-bonds of the simulation at $325 \mathrm{~K}$ reveal a transient period between 36 and $41 \mathrm{~ns}$. While composed of $\pi$-helix and $\mathrm{H}$ bonded turns during the dominant period, configurations of residues Leu19 to Asn 21 consist almost exclusively of H-bonded turns in the transient period. The residues Glu22 to Lys 28 evidence a change from configurations of $\alpha$ - and $\pi$-helices in the dominant period to configurations of almost pure $\alpha$-helix in the transient period. Interactions involving backbone-to-side-chain H-bonds between His18 and Glu20/ Asn21 (21NH-18ND1, 20NH-18ND1) and between Tyr17/His18 (17NH, 18NH) and Glu22 (22OE1 and 22OE2) occur in at least $90 \%$ of all configurations. These bonds constitute the dominant component of a loop structure, which is evident in three central members of the most-populated clusters shown in Fig. 4. The structural motif is reminiscent of capping boxes frequently found at the N-termini of $\alpha$-helices in globular proteins [31].

In the conformational cluster analysis, simulations of GCN4p16-31 at both 278 and $325 \mathrm{~K}$ showed convergence in the number of clusters after $20 \mathrm{~ns}$ using as cluster criterion that the backbone atom-positional RMSD be less than $0.10 \mathrm{~nm}$. The middle panel of Fig. 3 shows the temporal evolution of the ten most-populated clusters at $325 \mathrm{~K}$ ordered according to population; the analysis yielded a total of 78 clusters. The ten most-populated clusters include $93 \%$ of the configurations. Table 4 summarizes the analysis. Four clusters, i.e., 1, 6, 8 and 9, containing $68 \%$ of the configurations compose the dominant conformation. These clusters differ markedly only in the H-bonds and secondary structure of the residues between Lys28 and Gly31, and transitions from the sixth, eighth, and ninth clusters proceed almost exclusively to the first cluster. The initial $\alpha$-helical configurations, representing $6 \%$, are contained in the third cluster. The fourth and tenth clusters, corresponding to the $\alpha$-helical transient period, contain $6 \%$; transitions from the tenth cluster proceed almost exclusively to the fourth. In addition to these clusters, two other periods are represented among the first ten clusters. In the second and fifth clusters, representing $11 \%$ of the configurations, the $i, i+5 \mathrm{H}$-bonds between Ala24 and Leu29, and between Arg25 and Val30 present in the dominant cluster are broken. The second cluster contains configurations at times before the dominant configuration emerges, and transitions between it and the first cluster occur intermittently during the entire simulation, whereas the fifth emerges only towards the end of the $100 \mathrm{~ns}$. The seventh cluster represents the formation of $i, i+5 \mathrm{H}$-bonds between Val23 and His18, Ala24 and Leu19, Arg25 and Glu20, and between Leu26 and Asn21; no transitions proceed between it and any of the other ten most-populated clusters. The most likely lifetime for all clusters except the seventh was $5 \mathrm{ps}$, i.e., the resolution of the analysis, and the median lifetimes two to three times longer. The cluster and H-bond analysis of the peptide at $278 \mathrm{~K}$ are available as Supplementary Material $\left.^{1}\right)$. 


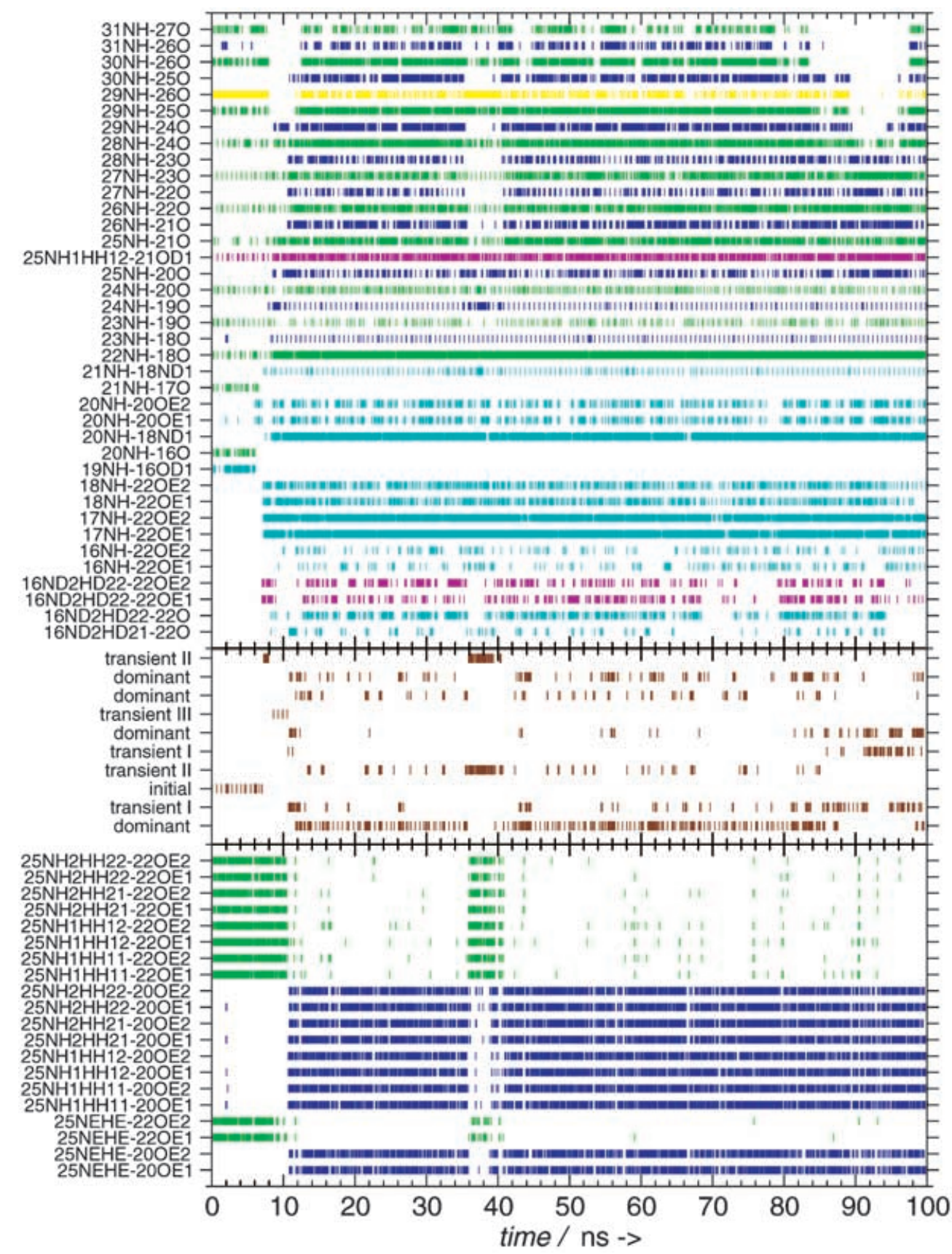

Fig. 3. Major H-bonds, conformational clusters, and salt bridges of GCN4p16-31 at $325 \mathrm{~K}$. For the H-bonds, green denotes $i, i+4$ bonds between backbone atoms; blue, $i, i+5$ bonds between backbone atoms, and yellow $i, i+3$ bonds between backbone atoms. Indigo denotes bonds between other backbone atoms, cyan denotes bonds between backbone atoms and side-chain atoms, and violet denotes those between side-chain atoms. Notation of the clusters is given in Table 4. For the salt bridges, defined by configurations in which the distance between oppositely charged atoms is less than $0.6 \mathrm{~nm}$, green denotes salt bridges between Arg25 and Glu22, and blue salt bridges between Arg25 and Glu20. 


\section{Initial (3)}

$31 \mathrm{G}$

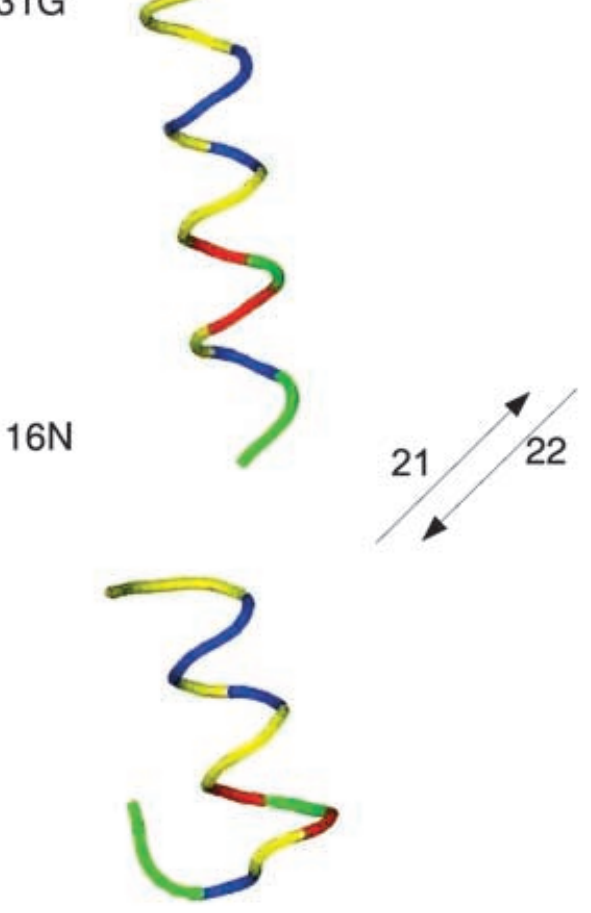

Transient II (4)
Dominant (1)

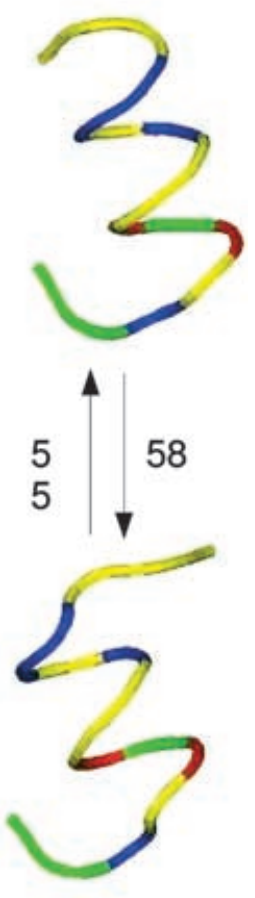

Transient I (2)

Fig. 4. Central conformations of the four major conformational clusters of GCN4p16-31. On the top left is the central conformation of the initial cluster, on the top right that of the dominant cluster, on the bottom left, that of the cluster transient I, the $\alpha$-helical transient period, and on the bottom right, the central conformation of the second-most-frequented cluster, transient III (see also Table 4). Negatively charged residues are designated by red, positively charged residues by blue. Yellow designates nonpolar and green polar residues. The numbers by

the arrows indicate the number of transitions between clusters in the direction indicated by the arrows.

A salt bridge between the charged residues Arg25 and Glu22 is observed in the crystal structure [20]; the lower panel of Fig. 3 displays the configurations in which intramolecular ionic interactions between side chains of the residues Arg25, Glu20, and Glu22 could occur using as criterion that oppositely charged atom pairs are separated by less than $0.6 \mathrm{~nm}$. Whereas the initial and transient regions, which exhibit $\alpha$-helical configurations, evidence ionic interactions between Arg25 and Glu22, the dominant region, characterized by $\pi$-helical configurations, evidences ionic interactions between Arg25 and Glu20. A similar analysis of the proximity of side chains of Lys27 or Lys28, and Glu20 or Glu22 evidenced no significant ionic interactions.

The simulation beginning with an extended structure at $278 \mathrm{~K}$ and $\mathrm{pH} 7.5$ shows two distinct regions. Between 14 and $46 \mathrm{~ns}$ form reciprocal H-bonds between the backbone atoms of residues Tyr17 and Val30 that appear correlated with $\beta$-bridge secondary 
Table 4. Conformational Cluster Analysis of the GCN4p16-31 at $325 \mathrm{~K}$ and $\mathrm{pH} 7.5$. The cluster criterion is a backbone atom-positional RMSD of less than $0.10 \mathrm{~nm}$. The clusters are grouped according to their conformational similarity as determined from analysis of H-bonds and transitions between groups. Columns 4 and 5 display the median and maximum lifetimes of the conformational clusters.

\begin{tabular}{rlrrr}
\hline Cluster & Period & Population [\%] & $\tau_{\text {med }}[\mathrm{ps}]$ & $\tau_{\text {max }}[\mathrm{ps}]$ \\
\hline 1 & dominant & 62 & 15 & 2525 \\
2 & transient I & 8 & 10 & 1155 \\
3 & initial & 6 & 30 & 1270 \\
4 & transient II & 5 & 10 & 255 \\
5 & transient I & 3 & 10 & 325 \\
6 & dominant & 3 & 10 & 125 \\
7 & transient III & 2 & 270 & 1465 \\
8 & dominant & 2 & 5 & 50 \\
9 & dominant & 1 & 5 & 25 \\
10 & transient II & 1 & 10 & 70 \\
\hline
\end{tabular}

structure in the two residues and with bends or H-bonded turns in neighboring residues. Bonds between backbone and side-chain atoms involving Leu29, Lys28, and His18 accompany these interactions. Analysis of salt bridges (not shown) reveals interactions between Lys 28 and Glu20. Like the H-bonds characterizing the first region, these salt bridges disperse after 52 ns. After 52 ns form numerous H-bonds between the sidechain atoms of Glu20 and backbone atoms of the neighboring residues, Glu22, Val23, and Ala24. Similar interactions involving Arg25 occur during the last $10 \mathrm{~ns}$ as well as bonds between backbone atoms of Gly31 and Tyr17, of Lys28 and Gly31, and of Leu19 and Gly31; these bonds appear correlated with the emergence of H-bonded turns and extended strands in the secondary structure. The almost uninterrupted appearance of the bend in the secondary structure of the three mid chain residues: Glu22, Val23, and Ala24, appears to be correlated with a salt bridge between Arg25 and Glu22 that populates $c a$. $14 \%$ of the configurations uniformly during the $100 \mathrm{~ns}$. Visual inspection shows that the peptide forms a loop during the entire simulation. The reciprocal $\mathrm{H}-$ bonds stabilize the loop in the first region of configuration, and bonds between Gly31, and Tyr17 or Leu19 stabilize it in the second. No helix formation is observed within the 100 -ns simulation. The analysis of RMSD and secondary structure are available as Supplementary Material $^{1}$ ).

GCN4p16-31 at pH 3 and in Salt Solution. Both at pH 3 and in salt solution, the initial structure endured for almost $20 \mathrm{~ns}$ with the RMS deviations fluctuating $c a$. $0.5 \mathrm{~nm}$. At pH 3 and $278 \mathrm{~K}$, the peptide sampled few $\pi$-helical conformations in a transient period between 20 and $30 \mathrm{~ns}$; after $30 \mathrm{~ns}$, the region between residues Tyr17 and Val23 resumed dominantly $\alpha$-helical secondary structure, while the region between residues Asn 24 and Leu29 evidenced bends or no secondary structure. Analysis of the $\mathrm{H}$-bonds confirmed these observations and revealed the formation of a $\mathrm{H}$-bond between the backbone atoms of Leu19 and Gly31, evidencing a loop structure, during the final $15 \mathrm{~ns}$.

After the initial $20 \mathrm{~ns}$, the peptide in salt solution at $278 \mathrm{~K}$ evolved into configurations of fluctuating secondary structures containing $\alpha$ - and $\pi$-helices similar to those dominating the peptide at $278 \mathrm{~K}$ and $\mathrm{pH} 7.5$. In the final $12 \mathrm{~ns}$ of the simulation, the residues between Tyr17 and Glu22 evolved into mixtures of H-bonded turns and 
$3_{10^{-}}$or $\pi$-helices, or no structure. Mixed secondary structures of H-bonded turns, $\alpha$ - and $\pi$-helices evolved in residues between Val23 and Lys28, and the residues between Leu29 and Gly31, lost structure. The analysis of secondary structure and H-bonds in both conditions are available as Supplementary Material ${ }^{1}$ ).

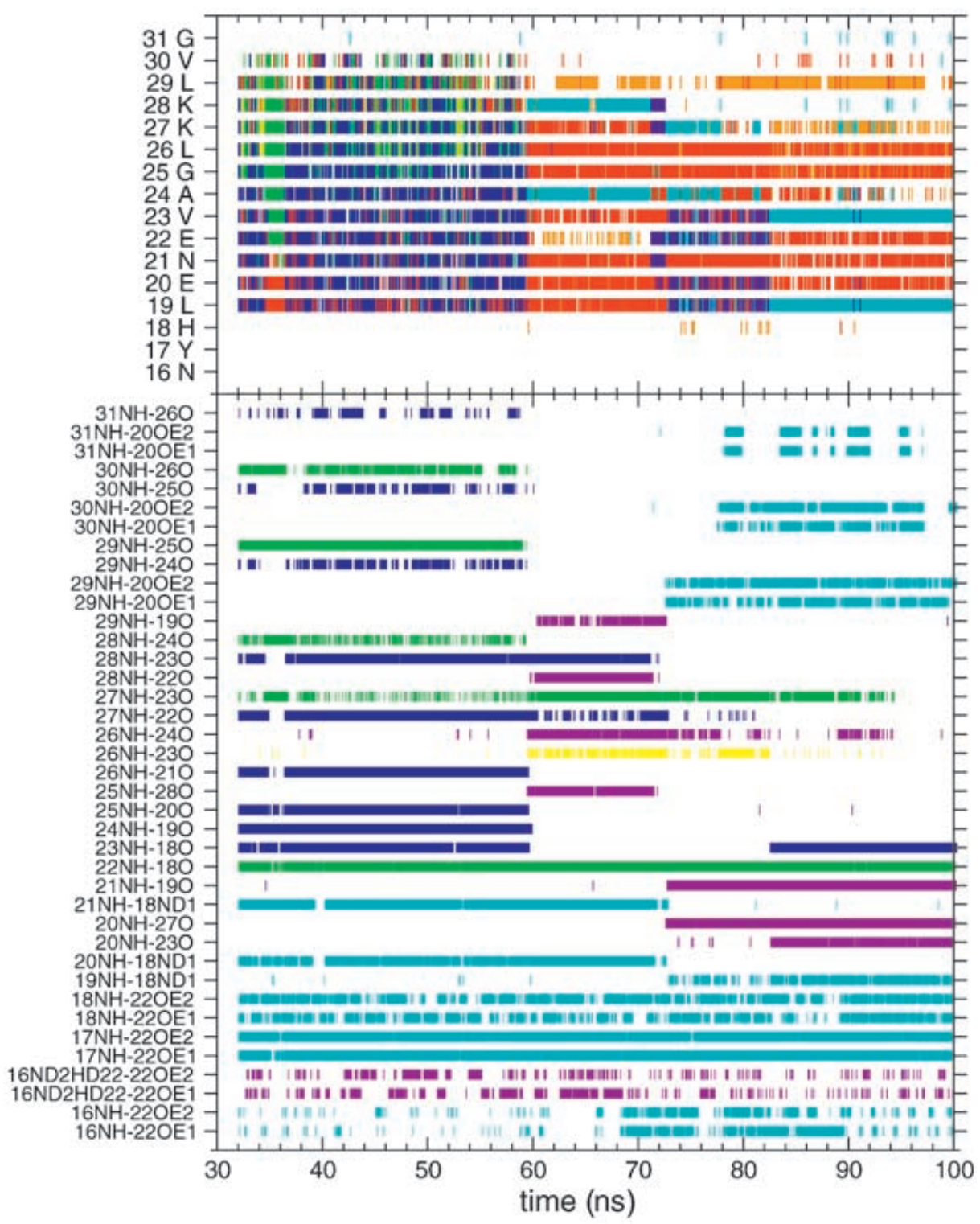

Fig. 5. Secondary structure and major H-bonds as a function of time for GCN4p16-31R25G at $325 \mathrm{~K}$. Notations for secondary structure are as in Fig. 1; the color code for H-bonds is as in Fig. 3. 
GCN4p16-31R25G. The mutant, in which a glycine residue was substituted for Arg25 at $325 \mathrm{~K}$, was started in the configuration of the GCN4p16-31 at $32 \mathrm{~ns}$, prior to the $5 \mathrm{~ns}$ transient period. As the secondary structures of Fig. 5 show, the simulation evolves in the first $27 \mathrm{~ns}$ in a manner similar to GCN4p16-31 at $325 \mathrm{~K}$; the transient period appears at about the same time point ( $34 \mathrm{~ns})$ but lasts less than $2 \mathrm{~ns}$, and the propensity for $\alpha$-helices increases in residues between Gly25 and Lys 28 . After $c a$. $27 \mathrm{ns,}$ the mutant unfolded suddenly to mixed configurations composed of bends, H-bonded turns, $\beta$-bridges, and extended strands. Analysis of the H-bonds reveals a cooperative dissolution of $i, i+5$ and $i, i+4$ bonds between backbone atoms of all but three residues. Bonds between side-chain atoms of Glu20 and the backbones of Leu29, Val30, and Gly31 form at $c a .75 \mathrm{~ns}$, as do bonds between backbone atoms of 19Leu and 21Asn, and of Glu20 and Lys27.

GCN4p2-17. As Fig. 6 shows, the N-terminal peptide at $278 \mathrm{~K}$ experienced within $1 \mathrm{~ns}$ a structural shift for a period lasting $26 \mathrm{~ns}$. Characterized by H-bonded turns or no structure in terminal residues, $\alpha$-helical secondary structure predominated in residues between Glu6 and Ser14. The distribution of RMS deviations during this period exhibits deviations of $0.05 \mathrm{~nm}$ about a central value of $0.15 \mathrm{~nm}$. Cluster analysis with a similarity criterion of $0.10 \mathrm{~nm}$ assigned nine of the ten most-populated clusters to this period.

At $26 \mathrm{~ns}$, the peptide experiences a sudden transition to predominantly $\pi$-helical secondary structure in residues between Leu5 and Asn16. In this second period, deviations of the RMSD distribution diminished to $0.01 \mathrm{~nm}$ about a central value of $0.30 \mathrm{~nm}$. Cluster analysis with a similarity criterion of $0.10 \mathrm{~nm}$ yielded a single cluster, which was associated with the formation of $i, i+5 \mathrm{H}$-bonds between backbone atoms of residues between Gln 4 and Ser14; analysis of $\mathrm{H}$-bonds showed that $i, i+4$ bonds were also present.

At $325 \mathrm{~K}, \mathrm{GCN} 4 \mathrm{p} 2-17$ proceeds after less than $2 \mathrm{~ns}$ to mixed secondary structures of $\alpha$ - and $\pi$-helices in residues between Gln 4 and Lys15. As Fig. 7 shows, the RMSD distribution is similar to that of the peptide at $278 \mathrm{~K}$, but the peak and the right tail are broader. The excursions reflect the disappearance between 5 and 12 ns of secondary structure in residues Ser14, Lys15, and Asn16, and the occurrence of transient periods between 16 and $20 \mathrm{~ns}$, and between 55 and $56 \mathrm{~ns}$ dominated by $\alpha$-helical secondary structure. Analyses of $\mathrm{H}$-bonds and clusters reveal that the dominant cluster describes $66 \%$ of the configurations. It is determined by $i, i+5 \mathrm{H}$-bonds between the backbone atoms of the residue pairs: Lys8-Lys3, Val9-Gln4, Glu10-Leu5, Glu11-Glu6, Leu12Asp7, Leu13-Lys8, Ser14-Val9, Lys15-Glu10, and Asn16-Glu11. The second, fifth, and ninth clusters, describing together $10 \%$ of the configurations, occur when the latter two of these pairs are broken. Breaking of the first three pairs determines the third cluster, which represents $5 \%$ of the configurations. The fourth cluster contains configurations, $4 \%$ of the total, associated with the $\alpha$-helical transient periods in which all $i, i+5 \mathrm{H}$ bonds, with the partial exception of the first pair, are broken. Thus, the time courses of the peptides at both temperatures are determined mainly by the formation and dissolution of the $i, i+5 \mathrm{H}$-bonds. Analysis of salt bridges reveals the prevalence at $325 \mathrm{~K}$ of three salt bridges: Lys15-Glu11, Lys3-Glu6, and Lys15-Glu10. The first persists during the entire simulation, whereas the second disperses for $10 \mathrm{~ns}$ during occupation of the third cluster. The third salt bridge also persists during the entire simulation, but is 


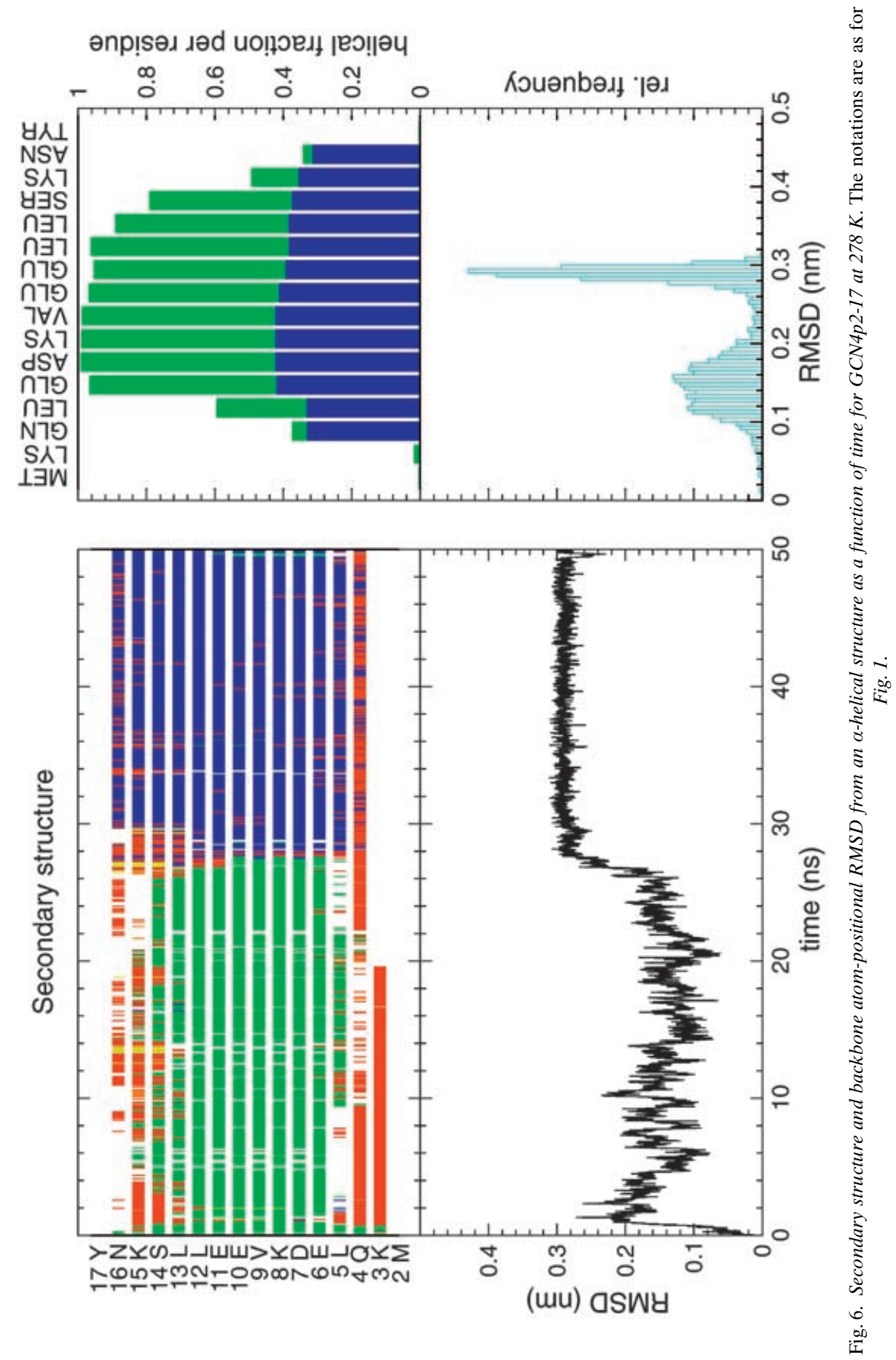




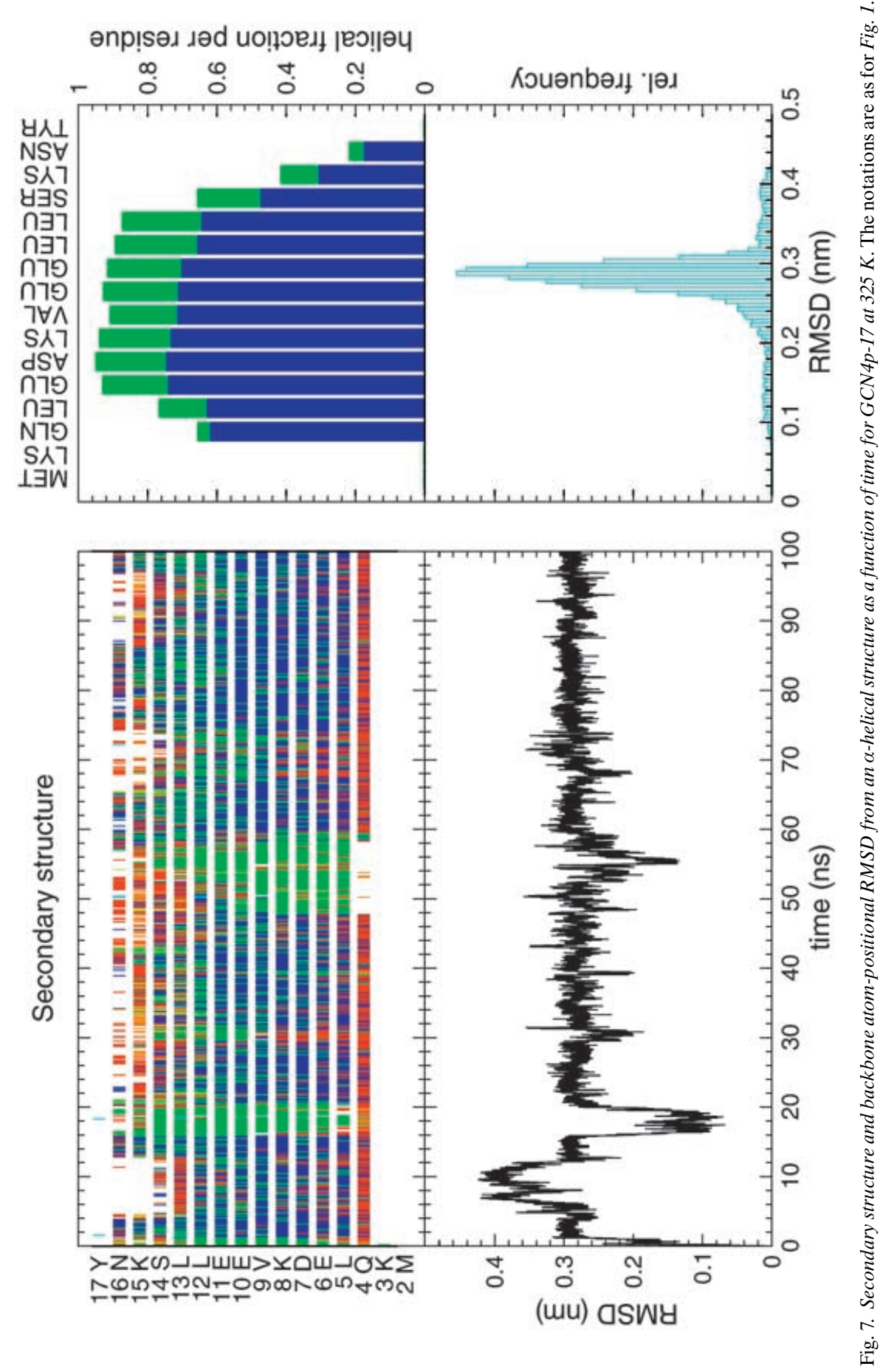


populated only about half as frequently $(23 \%)$ as the first salt bridge $(55 \%)$; the two bridges can occur simultaneously. At $278 \mathrm{~K}$, the first two salt bridges occur with frequencies of $c a .1 \%$, whereas the third occurs about a tenth as frequently; the mostprevalent salt bridge, Lys8-Glu11 populates $c a$. $8 \%$ of the configurations. The analyses of clusters and H-bonds at both temperatures are available as Supplementary Material $\left.^{1}\right)$.

Discussion. - Intended to explore mechanisms of coiled-coil formation, the C- and $\mathrm{N}$-terminal halves of the monomer found in the leucine zipper of GCN4 have been investigated with respect to their secondary structure under several conditions of temperature, $\mathrm{pH}$, and salt concentration by means of $\mathrm{CD}$. The NMR experiment presented above provides additional evidence about the $\mathrm{C}$-terminal half at $278 \mathrm{~K}$. To elucidate the experimental studies, we performed several MD simulations of the C-and $\mathrm{N}$-terminal halves of GCN4-p1 with explicit solvent under conditions representing the extremes of the experiments.

Both $\mathrm{C}$ - and N-terminal halves retained helical structure for the entirety of the 50ns simulations at $278 \mathrm{~K}$, and the 100 -ns simulations at $325 \mathrm{~K}$. The four simulations exhibited transitions between $\alpha$-helical secondary structure and dominantly $\pi$-helical secondary structure within $10 \mathrm{~ns}$ in three cases, and after $25 \mathrm{~ns}$ for the $\mathrm{N}$-terminal half at 278 K. Analyses of RMSD, secondary structure, H-bonds, and clusters revealed few distinctive differences between simulations of the $\mathrm{C}$ - and $\mathrm{N}$-terminal halves under the same conditions. In comparison, the $\mathrm{CD}$ experiments indicate marked helical secondary structure only in the C-terminal half at $278 \mathrm{~K}$. Simulations of the C-terminal half at pH 3 and in salt solution at $278 \mathrm{~K}$ do indicate incomplete loss within $50 \mathrm{~ns}$ of $\alpha$ helical secondary structure, consistent with the reduced helical propensity observed in CD experiments. A plausible explanation for the apparent stability of helical secondary structure in the first four simulations is insufficient simulation time to observe unfolding. A second factor in the evolution of the $\mathrm{N}$-terminal half is the existence of salt bridges that prolong the relaxation time. The limitation on simulation time is also indicated by the simulation of the folding of the extended C-terminal half, which does not acquire helical secondary structure within $100 \mathrm{~ns}$. Simulations lasting two to ten times longer might be necessary to reveal these differences and to observe folding of the extended C-terminal half.

The persistence of helical configurations in the residues 16-21 of GCN4p16-31 at $278 \mathrm{~K}$ yields values of $\mathrm{H}_{\mathrm{N}}(i)-\mathrm{H}_{\mathrm{N}}(i+1)$ NOE distances and ${ }^{3} J\left(\mathrm{H}_{\mathrm{N}}, \mathrm{H}_{\mathrm{C}_{\alpha}}\right)$ coupling constants which deviate from the experimental values and statistical predictions by the program AGADIR [32]. The latter indicate a pattern of helical propensity that is small between residues 16 and 21, obtains a high plateau between residues 22 and 28, and then decreases. Distances and coupling constants for the remaining residues $22-30$ are consistent with the data and predictions within the statistical uncertainties of the simulations. The simulations of the distances or coupling constants show little dependence on temperature.

The observation of $\pi$-helical configurations in numerous simulations of peptides has recently become a subject of dispute. Although rarely encountered, $\pi$-helices have been identified as unique structural elements in a variety of proteins [33][34]. Fodje and Al-Karadaghi have argued that the frequency of $\pi$-helices observed in data banks is 
an underestimate, and showed that reasonably modified criteria increase the frequency by more than an order of magnitude [33] Brooks and co-workers have argued that their occurrence is an artifact of the force fields used [35].

Experimental evidence is not conclusive. The measure of helical propensity provided by $\mathrm{CD}$ data does not discriminate between $\alpha$ - and $\pi$-helical configurations, and the analysis of NOEs and ${ }^{3} J$-coupling constants deduced from the NMR measurements indicates that the pure $\alpha$-helical configuration present in the initial cluster in the simulations of GCN4p16-31 at $325 \mathrm{~K}$ cannot be distinguished from the $\pi$ helical configurations in the dominant cluster.

Furthermore, X-ray crystallography of the dimer of the complete 33-residue GCN4p1 yielded the $\alpha$-helical starting configurations of the monomeric $\mathrm{C}$ - and $\mathrm{N}$-terminal halves. Dimerization might have favored this configuration, producing a more-compact structure than that of the monomer dissolved in $\mathrm{H}_{2} \mathrm{O}$. To our knowledge, $\alpha$ to $\pi$ transitions have never appeared in simulations, using the same (GROMOS) force field and of comparable duration, of globular proteins. This suggests that the packing of secondary structural elements hinders such transitions.

The pattern of $\pi$-helical configurations appearing in the simulations of the $\mathrm{C}$ - and $\mathrm{N}$-terminal halves is consistent with the one observed by Fodje and Al-Karadaghi [33]. Their recent statistical analysis revealed that $0.3 \%$ of all amino acids found in the Protein Data Bank are present in $\pi$-helices of 7 to 13 residues in length. The authors further found that aromatic and large aliphatic amino acid residues are generally preferred in $\pi$-helices relative to small ones. Accordingly, the sequence of GCN4-p1 might indeed have an intrinsic propensity for $\pi$-helix configurations. The salt-bridge analysis of the simulation of the C-terminal half at $325 \mathrm{~K}$ suggests that the electrostatic interaction between Arg25 and Glu20 constitutes a primary driving force for $\pi$-helix formation in this peptide. Salt-bridge analysis of the N-terminal half reveals that the electrostatic interaction between Lys15 and Glu10 plays a similar role in $\pi$-helix formation in this peptide. Simulations of the mutant of the C-terminal half, in which arginine was substituted by glycine at position 25 , demonstrate the loss of helical secondary structure when the charged residue is replaced. The simulations show, moreover, that the loss occurs suddenly and cooperatively with the dissolution of $i, i+5$ and $i, i+4 \mathrm{H}$-bonds associated with $\pi$ - and $\alpha$-helical configuration.

A central feature of the GCN4-p1 folding mechanism is the C-terminal trigger sequence [36]. This distinctive sequence motif was shown to act as an autonomous helical folding unit that limits the number of possible chain conformations prior to their encounter. As such, it provides an effective structural framework for the parallel inregister interaction of two helical trigger sequences early in the folding pathway [36][22-24]. A prediction emerging from the GCN4-p1 folding model is the existence of a signal sequence that prevents helix propagation of the $\mathrm{C}$-terminal half towards the $\mathrm{N}$-terminal half of the monomer. Capping interactions at helix boundaries have been shown to constitute such signals [30]. The simulations of GCN4p16-31 at $325 \mathrm{~K}$ revealed the stable formation of a complex network at the $\mathrm{N}$-terminus of the monomeric peptide involving both short- and mid-range interactions that indeed shows a helix cap signature (Figs. 3 and 4). The structural motif established by H-bonds of the His 18 and Glu22 side chains in combination with the salt bridges formed between the Glu20/Glu22 and Arg25 side chains differs from the one predicted by AGADIR [32]. 
This program predicts an N-terminal cap structure in which Asn21 acts as the Ncap residue instead of His18, and the Glu20 and Arg25 side chains establish a 'hydrophobic staple' [30]. The interaction pattern revealed by the simulation differs from the 'capping box' frequently found at the N-termini of $\alpha$-helices in globular proteins [31]. Typically, capping boxes are characterized by a reciprocal $\mathrm{H}$-bond pattern between the helical Ncap residue and the one situated three residues downstream in the helix.

Conclusions. - Apparent discrepancies between experimental observations and the simulations suggest that the relatively short simulation times hinder decisive comparison with experiment regarding stability and folding. However, the simulations suggest that the $\alpha$-helical structure determined by X-ray crystallography of the dimer GCN4-p1 does not represent the ensemble of structures populated by the GCN4p16-31 monomer; $\pi$-helical conformations appear to dominate the configurations. An additional feature of the simulations is the emergence of a distinctive network of $\mathrm{H}$ bonds reminiscent of a capping box. This network might represent a specific structural feature of peptides assuming $\alpha$ - and $\pi$-helical conformations. Moreover, the $\pi$-helix configuration might serve as a precursor to the zipping up that is an essential aspect of the kinetic model of GCN4-p1 coiled-coil formation.

The authors benefited from the advice and assistance of Indira Chandrasekhar, Chris Oostenbrink, Christine Peter, Alice Glättli, Markus Christen, Mika Kastenholz, and Tim Heinz. Financial support by the National Center of Competence in Research (NCCR) Structural Biology of the Swiss National Science Foundation (SNSF) is gratefully acknowledged.

\section{Experimental Part}

Simulation Setup. The eight MD simulations were performed using the GROMOS96 program modules and the 43A1 force field [36-38].

The initial coordinates of the $\mathrm{C}$ - and $\mathrm{N}$-terminal monomers in the $\alpha$-helical configuration were derived from the X-ray structure of the leucine zipper coiled coil obtained from the Protein Data Bank [20].

For each simulation, the solute was placed at the center of a periodic truncated octahedral box. The minimum distance from any atom of the peptide to the square box walls was chosen to be at least $1.4 \mathrm{~nm}$ in the initial configuration. The solvent, modeled according to SPC water [39], was introduced into the box by using a cubic periodic configuration of 216 pre-equilibrated $\mathrm{H}_{2} \mathrm{O}$ molecules. The minimum distance between an $\mathrm{O}$-atom of $\mathrm{H}_{2} \mathrm{O}$ and non-H-atoms of the solute was set to $0.23 \mathrm{~nm}$.

A steepest-descent energy minimization of the systems was performed to relax the solute-solvent contacts, while positionally restraining the solute atoms using a harmonic interaction with a force constant of $250 \mathrm{~kJ}$ $\mathrm{mol}^{-1} \mathrm{~nm}^{-2}$. Next, steepest-descent energy minimization of the system without restraints was performed to eliminate any residual strain. The energy minimizations were terminated when the energy change per step became smaller than $0.1 \mathrm{~kJ} \mathrm{~mol}^{-1}$. The MD simulations were started by taking the initial velocities from a Maxwell distribution at $100 \mathrm{~K}$. Solvent and solute were independently weakly coupled to a temp. bath with a relaxation time of $0.1 \mathrm{ps}$ [40]. The systems were also coupled to a pressure bath at $1 \mathrm{~atm}$ with a relaxation time of $0.5 \mathrm{ps}$ and an isothermal compressibility of $0.4575 \times 10^{-3}\left(\mathrm{~kJ} \mathrm{~mol}^{-1} \mathrm{~nm}^{-3}\right)^{-1}$ [39]. Bond lengths were constrained using the SHAKE algorithm with a geometric tolerance of $10^{-4}$ [41], so that the leapfrog integration time step could be set to 0.002 ps. For the non-bonded interactions a triple-range method with cutoff radii of $0.8 \mathrm{~nm}$ and $1.4 \mathrm{~nm}$ was used. Outside the longer cutoff radius, a reaction-field approximation was used with a relative dielectric permittivity of 66.6 [42]. Short-range Van der Waals and electrostatic interactions were evaluated at every time step by using a charge-group pair-list. Long-range Van der Waals and electrostatic interactions, between pairs at a distance longer than $0.8 \mathrm{~nm}$ and shorter than $1.4 \mathrm{~nm}$, were evaluated every fifth time step, at which point the pair list was updated.

Analysis. Trajectory coordinates and energies were stored at 0.5 -ps intervals and used for analysis. Analysis procedures comprised: 
Atom-Positional RMSD: least-squares translational and rotational fitting of atomic configurations and calculation of atom-positional root-mean-square differences (RMSD) between atomic configurations was based on the $\mathrm{N}$-, $\mathrm{C}_{\alpha^{-}}, \mathrm{C}$-, $\mathrm{O}$-atoms of all but the $\mathrm{N}$ - and $\mathrm{C}$-terminal residues of the peptides.

Secondary Structure: Assignment at 10-ps intervals according to the method of [43] using the program PROCHECK [29].

$H$-Bonds: Defined by a minimum donor- $\mathrm{H}$ - acceptor angle of $135^{\circ}$ and a maximum $\mathrm{H}$-acceptor distance of $0.25 \mathrm{~nm}$, H-bond percentages were calculated on the entire set of structures taken at 0.5 -ps intervals.

Conformational Cluster Analysis: A cluster analysis of peptide conformations 5 ps apart was performed on all trajectories from the simulations. The clustering algorithm, which uses the atom-positional RMSD as similarity criterion, has been described in previous studies of peptide dynamics [44]; it performs a rotational and translational least-squares fit for every pair of configurations using all backbone atoms but the N- and Cterminal residues of the peptides, and calculates the corresponding atom-positional RMSD for the same set of atoms. The similarity criteria used depended on the convergence of the simulations with respect to the RMSD and the number of clusters as a function of simulation time. The average lifetimes of particular conformers, corresponding to clusters of structures, were calculated from the time sequence of the sampling of clusters. Every time a particular cluster is abandoned, the time that the peptide has spent in this cluster is saved and used for the calculation of the average lifetime of the conformer.

$N M R$ : Interproton distances in the simulations were compared to the experimental $\mathrm{H}_{\mathrm{N}}(i)-\mathrm{H}_{\mathrm{N}}(i+1) \mathrm{NOE}$ intensities where $i$ denotes the residue sequence number. The former were calculated as $\left\langle r^{-6}\right\rangle^{-1 / 6}$, i.e., using $r^{-6}$ averaging over the trajectory structures, where $r$ indicates the actual proton-proton distance. ${ }^{3} J$-Coupling constants were calculated from the simulations using the Karplus relation,

$$
{ }^{3} J(\mathrm{H}, \mathrm{H})=a \cos ^{2} \theta+b \cos \theta+c
$$

where $a, b$, and $c$ were chosen equal to $6.4,-1.4$, and $1.9 \mathrm{~Hz}$, for calculating ${ }^{3} J\left(\mathrm{H}_{\mathrm{N}}, \mathrm{H}_{\mathrm{C}_{\alpha}}\right)[45]$.

NMR Experiments. All NMR experiments were carried out at $5^{\circ}$ on a Varian UnityPlus 600 spectrometer operating at 600-MHz. Spectra were recorded from 3 m M GCN4p16-31 peptide solns. in $10 \mathrm{~mm} \mathrm{NaCl,} 20 \mathrm{~mm} \mathrm{Na-}$ phosphate at $\mathrm{pH}$ 7.4. The following spectra were recorded: TOCSY (DIPSI mixing sequence, mixing time 60 ms), NOESY (mixing time $150 \mathrm{~ms}$ ), HSQC, HSQC-TOCSY, and P.E.COSY.

\section{REFERENCES}

[1] X. Daura, B. Jaun, D. Seebach, W. F. van Gunsteren, A. E. Mark, J. Mol. Biol. 1998, 280, 925.

[2] Y. Duan, P. A. Kollman, Science 1998, 282, 740.

[3] M. Schäfer, C. Bartels, M. Karplus, J. Mol. Biol. 1998, 284, 835.

[4] M. Takano, T. Yamato, J. Higo, A. Suyama, K. Nagayama, J. Am. Chem. Soc. 1999, 121, 605.

[5] G. Hummer, A. E. Garcia, S. Garde, Proteins: Struct., Funct., Genet. 2001, $42,77$.

[6] C. Simmerling, B. Strockbine, A. E. Roitberg, J. Am. Chem. Soc. 2002, 124, 11258.

[7] C. D. Snow, H. Nguyen, V. S. Pande, M. Gruebele, Nature 2002, 420, 102.

[8] M. Prévost, I. Ortmans, Proteins: Struct., Funct., Genet. 1997, 29, 212.

[9] A. R. Dinner, T. Lazaridis, M. Karplus, Proc. Natl. Acad. Sci. U.S.A. 1999, 96, 9068.

[10] V. S. Pande, D. S. Rokhsar, Proc. Natl. Acad. Sci. U.S.A. 1999, 96, 9062.

[11] D. Roccatano, A. Amadei, A. Di Nola, H. J. C. Berendsen, Protein. Sci. 1999, 8, 2130.

[12] A. M. J. J. Bonvin, W. F. van Gunsteren, J. Mol. Biol. 2000, 296, 255.

[13] P. Ferrara, A. Caflisch, Proc. Natl. Acad. Sci. U.S.A. 2000, 97, 10780.

[14] B. Y. Ma, R. Nussinov, J. Mol. Biol. 2000, 296, 1091.

[15] H. W. Wang, S. S. Sung, J. Am. Chem. Soc. 2000, 122, 1999.

[16] X. Daura, K. Gademann, H. Schäfer, B. Jaun, D. Seebach, W. F. van Gunsteren, J. Am. Chem. Soc. 2001, $123,2393$.

[17] J. Higo, O. V. Galzitskaya, S. Ono, H. Nakamura, Chem. Phys. Lett. 2001, 337, 169.

[18] G. Colombo, D. Roccatano, A. E. Mark, Proteins: Struct., Funct., Genet. 2002, 46, 380.

[19] X. Wu, S. Wang, B. R. Brooks, J. Am. Chem. Soc. 2002, 124, 5282.

[20] E. K. O'Shea, J. D. Klemm, P. S. Kim, T. Alber, Science 1991, 254, 539.

[21] R. A. Kammerer, T. Schultess, R. Landwehr, A. Lustig, J. Engel, U. Aebi, M. O. Steinmetz, Proc. Natl. Acad. Sci. U.S.A. 1998, 95, 13419. 
[22] J. K. Myers, T. G. Oas, J. Mol. Biol. 1999, 289, 205.

[23] L. B. Moran, J. P. Schneider, A. Kentsis, G. A. Reddy, T. R. Sosnick, Proc. Natl. Acad. Sci. U.S.A. 1999, 96, 10699.

[24] J. A. Zitzewitz, B. I. Ibarra-Molero, D. R. Fishel, K. L. Terry, C. R. Matthews, J. Mol. Biol. 2000, 296, 1105.

[25] W. F. van Gunsteren, A. E. Mark, J. Chem. Phys. 1998, 108, 6109.

[26] L. J. Smith, X. Daura, W. F. van Gunsteren, Proteins 2002, 48, 487.

[27] M. Gruber, A. N. Lupas, Trends Biochem. Sci. 2003, 28, 679.

[28] R. A. Kammerer, V. A. Jaravine, S. Frank, T. Schultess, R. Landwehr, A. Lustig, J. Engel, C. GarciaEcheverria, A. T. Alexandrescu, M. O. Steinmetz, J. Biol. Chem. 2001, 276, 13685.

[29] L. J. Smith, K. A. Bolin, H. Schwalbe, M. W. MacArthur, J. M. Thornton, C. M. Dobson, J. Mol. Biol. 1996, $255,494$.

[30] R. A. Laskowski, M. W. MacArthur, D. S. Moss, J. M. Thornton, J. Appl. Crystallogr. 1993, 26, 283.

[31] R. Aurora, G. D. Rose, Protein Sci. 1998, 7, 21.

[32] E. Lacroix, A. R. Viguera, L. Serrano, J. Mol. Biol. 1998, 284, 173.

[33] M. N. Fodje, S. Al-Karadaghi, Protein Eng. 2002, 15, 353.

[34] T. M. Weaver, Protein Sci. 2000, 9, 201.

[35] M. Feig, A. D. MacKerell Jr., C. L. Brooks III, J. Phys. Chem. B 2003, 107, 2831.

[36] W. F. van Gunsteren, S. R. Billeter, A. A. Eising, P. H. Hünenberger, P. Krüger, A. E. Mark, W. R. P. Scott, I. G. Tironi, 'Biomolecular simulation: The GROMOS96 manual, user guide', Vdf Hochschulverlag AG an der ETH-Zürich, Zürich, Switzerland, 1996.

[37] P. H. Hünenberger, W. F. van Gunsteren, 'Empirical Classical Force Fields for Molecular Systems', in 'Potential Energy Surfaces, Proceedings of the Mariapfarr Workshop in Theoretical Chemistry', Ed. A. F. Sax, Springer-Verlag, Berlin, 1999, p. 178

[38] X. Daura, W. F. van Gunsteren, A. E. Mark, Proteins 1999, 34, 269.

[39] H. J. C. Berendsen, J. P. M. Postma, W. F. van Gunsteren, J. Hermans, 'Interaction models for water in relation to protein hydration', in 'Intermolecular Forces', Ed. B. Pullman, Reidel, Dordrecht, 1981, p. 331.

[40] H. J. C. Berendsen, J. P. M. Postma, W. F. van Gunsteren, A. DiNola, J. R. Haak, J. Chem. Phys. 1984, 81, 3684.

[41] J.-P. Ryckaert, G. Ciccotti, H. J. C. Berendsen, J. Comput. Phys. 1977, 23, 327.

[42] A. Glättli, X. Daura, W. F. van Gunsteren, J. Chem. Phys. 2002, 116, 9811.

[43] W. Kabsch, C. Sander, Biopolymers 1983, 22, 2577.

[44] X. Daura, A. E. Mark, W. F. van Gunsteren, J. Comput. Chem. 1998, 19, 535.

[45] A. Pardi, M. Billeter, K. J. Wüthrich, J. Mol. Biol.1984, 180, 741. 